\title{
The State of Fatigue and Sleep among Clinical Nurses in Japan
}

\author{
Naomi Sumi' ${ }^{1}$ Naotaka Sugimura ${ }^{2}$, Yuko Yoshida', Rika Yano ${ }^{1}$ \\ ${ }^{1}$ Faculty of Health Sciences, Hokkaido University, Sapporo, Japan \\ ${ }^{2}$ Graduate School of Health Sciences, Hokkaido University, Sapporo, Japan \\ Email: nsumi@hs.hokudai.ac.jp
}

How to cite this paper: Sumi, N., Sugimura, N., Yoshida, Y. and Yano, R. (2017) The State of Fatigue and Sleep among Clinical Nurses in Japan. Open Journal of Nursing, 7, 1493-1501.

https://doi.org/10.4236/ojn.2017.712104

Received: November 21, 2017

Accepted: December 25, 2017

Published: December 28, 2017

Copyright (c) 2017 by authors and Scientific Research Publishing Inc. This work is licensed under the Creative Commons Attribution International License (CC BY 4.0).

http://creativecommons.org/licenses/by/4.0/

(c) (i) Open Access

\begin{abstract}
The objective of this study was to investigate the state of fatigue and sleep among clinical nurses in Japan. This descriptive cross-sectional study was conducted from December 2015 to January 2016. The participants were nurses who worked in public hospitals with 500 beds in the major cities and regional cities of Hokkaido. Fatigue was quantitatively assessed using the Cumulative Fatigue Symptoms Index (CFSI). Among nurses in their twenties, the rate of complaints about anxiety and decrease in vitality were high, which was replaced with complaints about irritability among nurses in their thirties. The most popular complaint among nurses in their forties was general fatigue. There was no difference in complaint ratios concerning the workplace location among nurses in their twenties and thirties, nurses in their forties working in suburban areas complained about fatigue more than their urban area. Nurses with sleep problems related to anxiety have a significantly higher complaint rate for all the eight items of CFSI compared with the nurses who do not have such problems $(p<0.001)$. This is an intermediate report and is part of a study that aims to develop a health management program for hospital nurses regarding fatigue and sleep.
\end{abstract}

\section{Keywords}

Hospital Nurse, Fatigue, Cumulative Fatigue Symptoms Index , Sleep

\section{Introduction}

Although the job separation rate for Japanese nurses is on the decline, in general it hovers around $11 \%$ and is $7.5 \%$ for new nurses [1]. The most commonly cited reason for nurses leaving their job is "female life events" such as childbirth and childcare, followed by physical health problems and psychological health prob- 
lems [2]. Previous studies on burnout among nurses and physical and psychological health suggest that chronic fatigue, depression, and other symptoms are correlated to burnout [3]. The results of a large-scale survey of nurses reported that fatigue interferes to the onset of emotional exhaustion and depersonalization [4]. Early intervention into nurse burnout can prevent job separation among nurses and therefore the issue of nurses maintaining and improving their own physical and mental health is an important issue that can lead to improved quality of healthcare. Since 2015, in Japan, the Industrial Safety and Health Act has required employers with at least 50 employees to provide worksite stress checks [5]. In addition, in 2013 the Japanese Nursing Association released its guidelines on night shifts and shift work [6], which includes educational contents related to sleepiness during nurses' shift work and provides guidelines regarding the taking of breaks. These efforts not only reflect the importance of aspects such as work management of nurses and organizational management of hospitals, but also the importance of nurses themselves making efforts to manage stress and their own physical and mental health in order to prevent burnout. Previous studies have assessed and shown the relation between sleep among nurses who work shifts and chronic disease, and sleep is thought to be a predictive index of chronic fatigue [7]. The relationship between the degree of sleep problems and fatigue is not fully considered. Therefore, in this study, we aimed to study the relationship between the current state of fatigue among Japanese nurses and sleep.

\section{Objective of the Study}

The purpose of this study was to elucidate the stages of fatigue among nurses in hospitals as well as factors related to sleep in order to obtain suggestions for stress and health management strategies that the nurses themselves can employ.

\section{Methodology}

This descriptive cross-sectional study was conducted from December 2015 to January 2016. The subjects were nurses who work in public hospitals in the major cities and regional cities of Hokkaido. Two hospitals were extracted from each group of urban and suburban cities using convenient sampling. Furthermore, in order to investigate the degree of fatigue of nurses in the present study, to eliminate the influence of differences in nurses' work details due to hospital functions, public hospital having 400 or more beds were used as the selection criteria. Since their normal work schedules-including night shift work-may differ, directors and deputy directors of nursing departments as well as head nurses were excluded. We included both nurses in outpatient and inpatient wards

The survey was an anonymous, self-administered drop-off survey. The survey description and questionnaire forms were distributed to the subjects through the nursing departments of the participating hospitals and they were recovered by the researcher from each hospital. The subjects were also guaranteed that their 
participation in the survey would be of their own free will. Consent was assumed when the subjects returned their completed questionnaire forms to the researcher.

We assessed fatigue using the Cumulative Fatigue Symptoms Index (CFSI), developed by Kosugoh [8]. The questionnaire comprises 81 items over eight categories in three clusters: mental symptoms (anxiety: 11 items; depressive feelings: 9 items; decreased vitality: 9 items); physical symptoms (general fatigue: 7 items; chronic fatigue syndrome: 8 items); and workplace environment dissatisfaction (irritability: 7 items; decreased willingness to work: 13 items). Each item may be answered "yes" or "no". We calculated an "average complaint rate" for each category by dividing the number of "yes" responses within that category by the total number of items in that category. The CFSI can clarify the presence

of chronic fatigue and examine it objectively, because it asks respondents about symptoms felt consistently over several days, rather than only at a given time. The questionnaire forms were purchased from the copyright-holding organization, in quantity equivalent to the number of nurses to whom they were distributed.

\subsection{Ethical Considerations}

This survey was conducted with the approval of the Institutional Review Board of the Graduate School of Health Sciences, Hokkaido University and the Institutional Review Boards of the participating facilities.

\subsection{Data Management and Analysis}

Descriptive statistics were analyzed using SPSS version 23.0 (IBM Corp., Armonk, NY, USA). Inferential statistics were used to examine the regional differences in CFSI complaint rate by age group using t-test, and the differences in CFSI complaint rates by age group (3 groups) were performed using a one-way analysis of variance. We created a radar chart on complaint rates of items (No. 9, $18,60,74)$ that inquire about sleep condition in the CFSI.

\section{Results}

A total of 1890 survey forms were distributed and 1509 were recovered. The 1461 forms that did not have invalid or missing responses were used for the analysis (recovery rate: $79.8 \%$, effective recovery rate: $96.8 \%$ ). The participants comprised 1335 women (91.8\%), 610 participants were in their twenties (41.8\%), 452 participants were in their thirties (30.9\%), and 399 were in their forties (27.3\%). Five hundred two participants were married (34.6\%), and 421 were raising children at the time of the survey (29.0\%). Our investigation of their work schedules indicated that 296 worked the normal day shift (20.4\%) and 1125 worked other shifts (76.3\%). Analysis of the geographical location of the subjects indicated that 1074 lived in cities (73.5\%) and 387 lived in regional cities $(26.5 \%)$ (Table 1). 
Table 1. Basic attributes of nurses. $(\mathrm{N}=1461)$

\begin{tabular}{cccc}
\hline & Item & $\mathrm{N}$ & $\%$ \\
\hline Sex (a) & Female & 1335 & 8.2 \\
& Male & 119 & 91.8 \\
& $20 \mathrm{~s}$ & 610 & 41.8 \\
& $30 \mathrm{~s}$ & 452 & 30.9 \\
Marital status (a) & 40s and older & 399 & 27.3 \\
& Unmarried & 950 & 65.4 \\
Engaged in childcare (a) & Married & 502 & 34.6 \\
& No & 1031 & 71.0 \\
Engaged in nursing care (a) & Yes & 421 & 29.0 \\
& No & 1338 & 91.8 \\
Years of experience as a nurse & Yes & 120 & 8.2 \\
No. of years at present position & Mean \pm SD & & $11.6 \pm 9.0$ \\
Work schedule & Mean \pm SD & & $3.7 \pm 3.5$ \\
& Day shift & 296 & 20.4 \\
& Other shifts & 1125 & 78.4 \\
& Other & 20 & 1.2 \\
\hline
\end{tabular}

(a): Indicates the frequency and rate of the respondents.

The nurses' CSFI complaint rate was far greater in the eight categories than among Japanese women health workers $(n=6010)$ reported by Kosugoh [9]. Among the eight categories, chronic fatigue syndromes were the highest in all ages in Figure 1. With regard to the difference in the CFSI complaint rate, as a result of a one-way analysis of variance, nurses in their $20 \mathrm{~s}$ had higher complaint rates of anxiety than other age groups (20s: 30s: $40 \mathrm{~s}=39.2: 35.5: 32.6, p<0.001$ ). Although significant differences for nurses in their 20 s and 30 s were noted, depression, the rate of decreased motivation for work, signs of chronic fatigue syndrome (20s: $40 \mathrm{~s}=40.6: 35.2, p<0.05)$, decreased desire to work (20s: $40 \mathrm{~s}=$ 32.4: 27.6, $p<0.05$ ), and chronic fatigue syndrome (20s: 40s $=61.9: 53.7, p<$ $0.001)$ significantly increased. Nurses in their thirties had a higher percentage of complaints of irritability than nurses in their twenties (20s: 30s $=25.1: 30.8, p<$ 0.01 ). Among nurses in their twenties, chronic fatigue syndrome, decreased vitality, anxiety, and depressed feeling were higher than in the other age groups. Irritability was higher among nurses in their thirties than other age groups. Among nurses aged 40 years and older, those who lived in suburban cities had higher complaint rates on the CSFI than nurses in urban areas for all items except for physical disorders. In other age groups, there was no significant difference in complaint rate on the CFSI between urban and suburban cities.

Regarding complaint rate of CFSI by nurses' sleep problems, nurses who answered "I often feel upset and can't sleep at night" $(\mathrm{n}=292)$ scored higher in the 

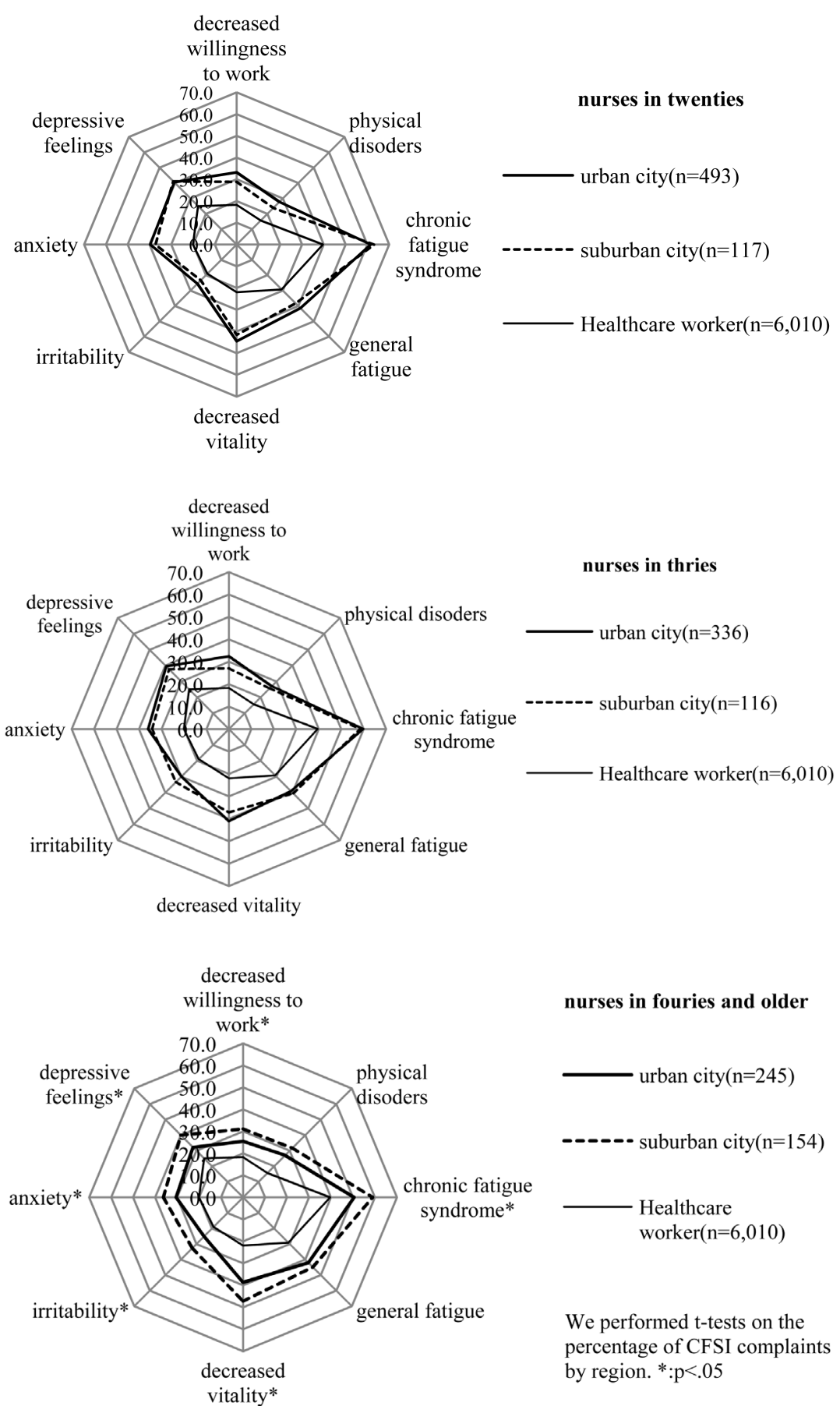

Figure 1. Compare of complaint rate of CFSI for nurses by age and work area with female Japanese health workers $(\mathrm{n}=6010)$.

eight categories of the CFSI compared to nurses with other sleep problems. Nurses who answered "I often feel upset and can't sleep at night" $(\mathrm{n}=382)$ had an increasing rate of complaints by CFSI (Figure 2). With respect to the four sleep problems, a $t$-test was used to compare the CFSI complaint rate of nurses who had problems with those who did not; for eight CFSI items, the complaint rate of nurses with problems was significantly higher $(p<0.001)$. Nurses with 


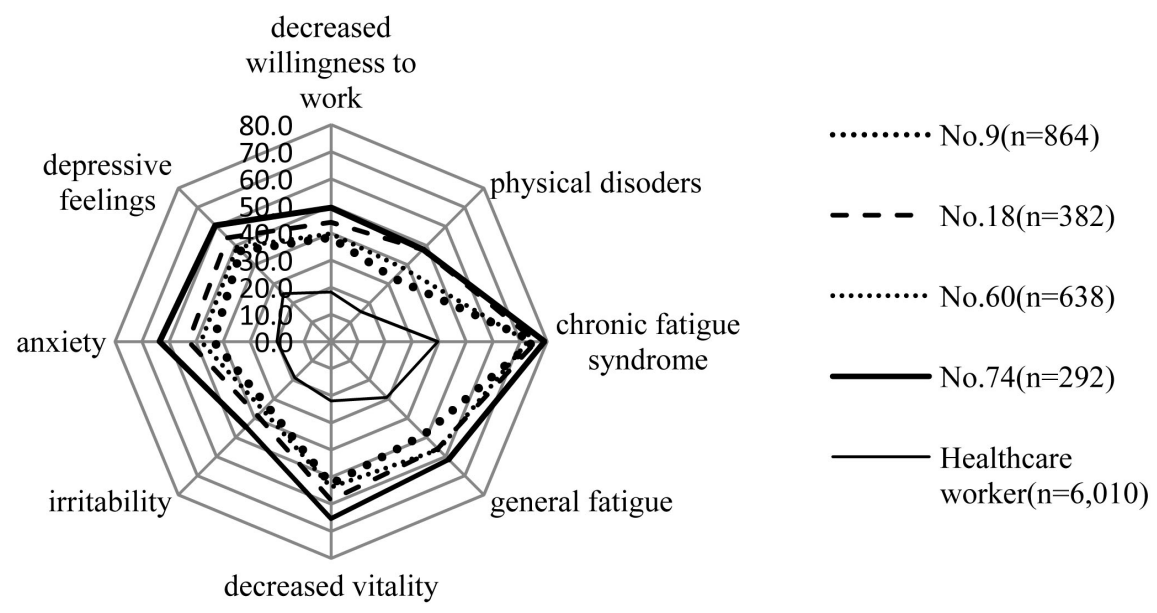

No.9, 18, 60, 74 are items that ask about sleep conditions in CFSI.

No.9: "I have lately felt sleepy every day"

No.18: "Recently, I can't easily fall asleep"

No.60: "I don't sleep well and often dream"

No.74: "I often feel upset and I can't sleep at night"

Figure 2. Comparison of CFSI results for nurses by sleep condition.

sleep problems related to anxiety in particular had a significantly higher complaint rate than nurses who did not [decreased willingness to work: 49.4 vs 25.8 , chronic fatigue syndrome: 79.0 vs 53.7 , physical dysfunction: 48.0 vs 21.9 , general fatigue: 61.6 vs 35.6 , decreased vitality: 65.4 vs 36.1 , irritability: 44.6 vs 23.2 , anxiety: 63.5 vs 29.2 , and depressive feelings: 60.7 vs $32.9(p<0.001)$.

\section{Discussion}

The participants of this study included more who were in younger age groups as compared to the statistics indicated in the Nurses' Employment Survey conducted by the Ministry of Health, Labor and Welfare in 2015 (twenties $=22 \%$, thirties $=31 \%$, forties and older $=47 \%)[10]$. One simple reason for the discrepancy is the fact that $90 \%$ of nurses in their 20 s are employed at hospitals. Another is that all facilities surveyed in the present study were large, cancer care district liaison hospitals having over 500 hospital beds; nurses with less than 5 years of working experience constitute approximately $30 \%$ of the nurses in such hospitals. Our data seems to reflect the relatively large contribution of this young age group to the age distribution.

Previous studies on fatigue among nurses have shown strong relationships between physical and emotional stress and fatigue symptoms [4]. Normal work can intensify into overwork when this regulation capacity is reduced. The CFSI results of nurses by age showed that nurses in their twenties have higher rate of CFSI than the other group and have high rates in emotional categories. Nurses in their 20s and 30s with family roles are particularly vulnerable; family roles such as child-rearing and their relationship with their spouse overlap with their careers [1]. It is inferred that nurses in their 40's may have multiple roles such as raising children and being caregivers for their parents [2]. These results are con- 
sistent with the finding of previous studies. Nurses are more likely to accumulate mental and physical fatigue when they cannot balance these roles. In the case of a nurse in her twenties, stress and fatigue lead to early unemployment. As a result, nurses in their twenties are complaining about anxiety, depression, fatigue, etc., and it is considered that there is a danger of fatigue. In a previous study, it is said that moderate burnout is related to physical symptoms whereas severe burnout is related to psychiatric symptoms [11]. It can be said that the risk of employment separation among novice and mid-level nurses is not based on regions, and it is necessary to have an awareness of fatigue, including anxiety and irritation as a factor influencing burnout. In the present study, nurses in their $40 \mathrm{~s}$ had a higher rate of complaint of fatigue than nurses in suburban areas. This is because, as an individual factor, in general, the nurses in suburban cities live with their family members rather than as a nuclear family. Therefore, in the case of nurses who live with family members in their 40 s, it is possible that fatigue may increase because of having to play multiple roles, such as childrearing and nursing care. Furthermore, as a hospital factor, the possibility of nurses' overwork due to a shortage of nurses in comparison with those in urban hospitals cannot be denied. Even if the employment separation rate is lower in urban than suburban areas, it is necessary to recognize that the risks of separation from employment and of deterioration of health disorders exist.

In this study, the difference in fatigue level became clear by the sleep pattern. The problem of sleep, particularly related to fatigue, reflects anxiety and enhancement of anxiety may cause mental problems [9]. It is not yet clear how the amount of sleep or a decrease in its quality promotes the feeling of fatigue [12]. It can be inferred from this result that sleep disorders associated with increased sense of anxiety may enhance fatigue and subsequently cause mental and physical problems. Therefore, screening the state of sleep among nurses may enhance our understanding of mental conditions, including anxiety and depression as well as fatigue. Therefore, it seems necessary to consider the relationship between factors such as fatigue, burnout, nurses' sleep, and so on. Future analysis is necessary to examine the relationships among factors such as nurse fatigue, burnout, and sleep.

\section{Conclusions}

We analyzed and reported on the relationships with burnout, physical and emotional fatigue and sleep. These results indicate that nurses of all age groups complained of chronic fatigue syndrome, while those in their 20s complained of anxiety and decreased vitality, and those in their 30s complained of irritability, suggesting that Japanese nurses are placed in a situation in which they are more likely to transition to a state of burnout. The difference in fatigue level became clear by the sleep pattern. As many nurses were found to experience intense fatigue, there is a need for increased awareness among nurses themselves concerning the changes in the physical condition and sleep-related problems and a need to promote nurse health management and measures to prevent burnout by 
both individuals and hospitals.

This study was a cross-sectional study targeting 4 relatively large-scale facilities in Hokkaido, and as a result its representativeness of the nursing population as a whole is limited. In addition, we did not investigate other factors such as work environment or human relations in this study. Therefore, it is essential to validate a burnout predictive index and assess nurses' fatigue and lifestyle including sleep condition

\section{Acknowledgements}

The authors would like to express their appreciation for the kind cooperation of all the nurses who participated in this study.

\section{Conflicts of Interest}

The authors have no conflicts of interest to declare.

\section{Research Funding}

This study was partially funded by a Ministry of Public Management, Home Affairs, Posts and Telecommunications SCOPE regional ICT promotion research and development grant (152301001) and was conducted as an empirical study for use in cloud services and the development of ICT for the purpose of the health management of nurses and the improvement of regional medical care.

\section{References}

[1] Japanese Ministry of Health, Labor and Welfare (2015) Actual Condition Survey on Hospital Nurses.

http://www.mhlw.go.jp/file/05-Shingikai-10801000-Iseikyoku-Soumuka/000007289 5.pdf

[2] Han, H. (2012) The Study on the Actual Conditions of the Nurse Shortage in Japan. Journal of East Asian Studies, 10, 1-24. (In Japanese)

[3] Arakawa, C. (2011) Factors Affecting the Resignation of Female Nurses: Longitudinal Study of Nurses working at Hospitals with more than 200 Beds in the General Ward in the Kanto Region. Japanese Journal of Nursing Research, 34, 85-92. (In Japanese)

[4] Raftopoulos, V., Charalambous, A. and Talias, M. (2012) The Factors Associated with the Burnout Syndrome and Fatigue in Cypriot Nurses: A Census Report. BMJ Public Health, 12, 457. https://doi.org/10.1186/1471-2458-12-457

[5] Japanese Ministry of Industry Safe and Health Act (2015) The Brief Job Stress Questionnaire.

http://www.mhlw.go.jp/file/06-Seisakujouhou-11300000-Roudoukijunkyokuanzene iseibu/0000181833.pdf

[6] Japanese Nursing Association (2013) Nurse's Guideline on Night Shift and Shift Work.

https://www.nurse.or.jp/nursing/shuroanzen/yakinkotai/guideline/index.htm

[7] Korompeli, A., Muurlink, O., Tzavara, C., et al. (2014) Influence of Shiftwork on Greek nursing Personnel. Safety and Health at Work, 5, 73-79.

https://doi.org/10.1016/j.shaw.2014.03.003 
[8] Kosugoh, R. (1991) Validity and reliability of Cumulative Fatigue Symptoms Index. Journal of Science of Labor, 67, 145-157. (In Japanese)

[9] Kosugoh, R., Fujii, H. and Hirata, A. (1992) Subjective Assessment of Workload (1) Revision of the Cumulative Fatigue Symptoms Index. Journal of Science of Labor, 68, 489-502. (In Japanese)

[10] Japanese Ministry of Health, Labor and Welfare (2015) Number of Nurses, Public Health Nurses, Associate Nurses. http://www.mhlw.go.jp/toukei/saikin/hw/eisei/14/dl/kekka1.p

[11] Narahara, R. and Shimada, R. (2012) Mental and Physical Symptoms Due to Degree of Burnout in Nurses Engaged in the Three-Shift Work: A Trial to Identify Burnout Symptoms Using the Japanese Version of GHQ 30 and of MBI. Journal of Health Sciences of Mind and Body, 8, 124-129. (In Japanese)

[12] Kagamiyama, H. and Yano, R. (2017) A Literature Review of Sleep among Nurses doing Shiftwork. Journal of Comprehensive Nursing Research, 18, 3-11. 